\title{
Cardiomyocyte-specific disruption of Cathepsin K protects against doxorubicin-induced cardiotoxicity
}

\author{
Rui Guo ${ }^{1}$, Yinan Hua ${ }^{1}$, Jun Ren (D)', Karin E. Bornfeldt ${ }^{2}$ and Sreejayan Nair (1)
}

\begin{abstract}
The lysosomal cysteine protease Cathepsin $\mathrm{K}$ is elevated in humans and animal models of heart failure. Our recent studies show that whole-body deletion of Cathepsin $\mathrm{K}$ protects mice against cardiac dysfunction. Whether this is attributable to a direct effect on cardiomyocytes or is a consequence of the global metabolic alterations associated with Cathepsin $\mathrm{K}$ deletion is unknown. To determine the role of Cathepsin $\mathrm{K}$ in cardiomyocytes, we developed a cardiomyocyte-specific Cathepsin K-deficient mouse model and tested the hypothesis that ablation of Cathepsin $\mathrm{K}$ in cardiomyocytes would ameliorate the cardiotoxic side-effects of the anticancer drug doxorubicin. We used an a-myosin heavy chain promoter to drive expression of $\mathrm{Cre}$, which resulted in over $80 \%$ reduction in protein and mRNA levels of cardiac Cathepsin K at baseline. Four-month-old control (Myh-Cre; Ctsk $^{\mathrm{fl} / \mathrm{fl}}$ ) and Cathepsin K knockout (Myh-Cre ${ }^{+}$; Ctsk ${ }^{\mathrm{fl} / \mathrm{fl}}$ ) mice received intraperitoneal injections of doxorubicin or vehicle, 1 week following which, body and tissue weight, echocardiographic properties, cardiomyocyte contractile function and $\mathrm{Ca}^{2}$

${ }^{+}$-handling were evaluated. Control mice treated with doxorubicin exhibited a marked increase in cardiac Cathepsin $\mathrm{K}$, which was associated with an impairment in cardiac structure and function, evidenced as an increase in endsystolic and end-diastolic diameters, decreased fractional shortening and wall thickness, disruption in cardiac sarcomere and microfilaments and impaired intracellular $\mathrm{Ca}^{2+}$ homeostasis. In contrast, the aforementioned cardiotoxic effects of doxorubicin were attenuated or reversed in mice lacking cardiac Cathepsin K. Mechanistically, Cathepsin K-deficiency reconciled the disturbance in cardiac energy homeostasis and attenuated NF-KB signaling and apoptosis to ameliorate doxorubicin-induced cardiotoxicity. Cathepsin K may represent a viable drug target to treat cardiac disease.
\end{abstract}

\section{Introduction}

Cathepsin $\mathrm{K}$ is a lysosomal cysteine protease, which has been widely studied in the context of osteoporosis ${ }^{1}$. Recently, reports suggest that activation of this protease may have a deleterious role in the pathophysiology of cardiometabolic diseases $^{2-4}$. Elevated levels of Cathepsin

\footnotetext{
Correspondence: Sreejayan Nair (sreejay@uwyo.edu)

${ }^{1}$ Center for Cardiovascular Research and Alternative Medicine, School of Pharmacy College of Health Sciences, University of Wyoming, Laramie, WY 82071, USA

UW Medicine Diabetes Institute, Departments of Medicine, Division of Metabolism, Endocrinology and Nutrition, and Pathology, School of Medicine, University of Washington, Seattle, WA 98109, USA
}

Edited by A. Stephanou
$\mathrm{K}$ have been demonstrated in both human and animal models of heart failure, atherosclerosis, and coronary heart disease ${ }^{5-7}$. Studies from our laboratory have revealed that global deletion of the Cathepsin $\mathrm{K}$ gene protects against cardiac dysfunction associated with pressure overload, diabetes, and aging ${ }^{8-11}$. However, it is unclear whether the beneficial effects of Cathepsin $\mathrm{K}$ knockout is attributable to its direct cardiac effects or a secondary effect consequent to improvement in wholebody metabolic changes. To determine whether Cathepsin $\mathrm{K}$ has a direct role in cardioprotection, we generated a conditional, cardiomyocyte-specific, Cathepsin K knockout mouse model. 
Doxorubicin is a potent and effective broad-spectrum antineoplastic anthracycline antibiotic used to treat a variety of cancers. However, the clinical use of doxorubicin is limited by its cardiotoxicity that leads to cardiomyopathy and heart failure. Doxorubicin-induced cardiotoxicity is cumulative and irreversible, and manifests as unfavorable cardiac morphologic and functional changes, including dilated cardiac chambers, impaired left ventricular contractility, reduced ejection fraction, reduced cardiac output, and associated diastolic dysfunction, as well as histopathologic alterations ${ }^{12}$. Previous studies have shown that doxorubicin-induced cardiotoxicity can be attributed to intracellular calcium disturbance, nucleic interruption and damage, impaired cardiac energy homeostasis, apoptosis, and oxidative stress resulting either from increased levels of reactive oxygen species and lipid peroxidation or reduced levels of antioxidants and sulfhydryl groups ${ }^{13-15}$.

In this study, we evaluated the effects of cardiomyocytespecific Cathepsin K-deficiency on doxorubicin-induced cardiac dysfunction. To understand the molecular mechanisms, we also evaluated the metabolic, apoptotic and inflammatory signaling.

\section{Results}

Generation and characterization of cardiac-specific

Cathepsin K knockout (Ctsk-CKO) mice

Mice with cardiomyocyte-targeted deletion of Cathepsin $\mathrm{K}$ were generated by utilizing ES cells carrying $F R T$ and loxP sites flanking Ctsk exon 2-5 that encodes the active site of Cathepsin K (Fig. 1a). Expression of Cre recombinase under control of the myh6 promoter results in deletion of exons 2-5 of Ctsk in the cardiomyocytes (Fig. 1b, c). Genotyping showed a LacZ positive band of $77 \mathrm{bp}$ (Fig. 1d); FLP mutant band of $340 \mathrm{bp}$, wild-type band of $650 \mathrm{bp}$ (Fig. 1e); and Myh-Cre mutant band of $300 \mathrm{bp}$ with an internal control band of $200 \mathrm{bp}$ (Fig. 1f). Ctsk mRNA levels were analyzed in left ventricles of control and Ctsk CKO mice using quantitative real-time PCR. Levels of Ctsk mRNA were reduced by $86.5 \%$ using primers targeting exon 2 and by $84.2 \%$ using primers targeting its coding region in Ctsk-CKO mice, as compared with littermate controls (Supplementary Figure 1A-B). Western blot analysis confirmed reduction in Cathepsin $\mathrm{K}$ protein levels in hearts from Ctsk-CKO mice, as compared with littermate control mice (Supplementary Figure 1C-D). There were no

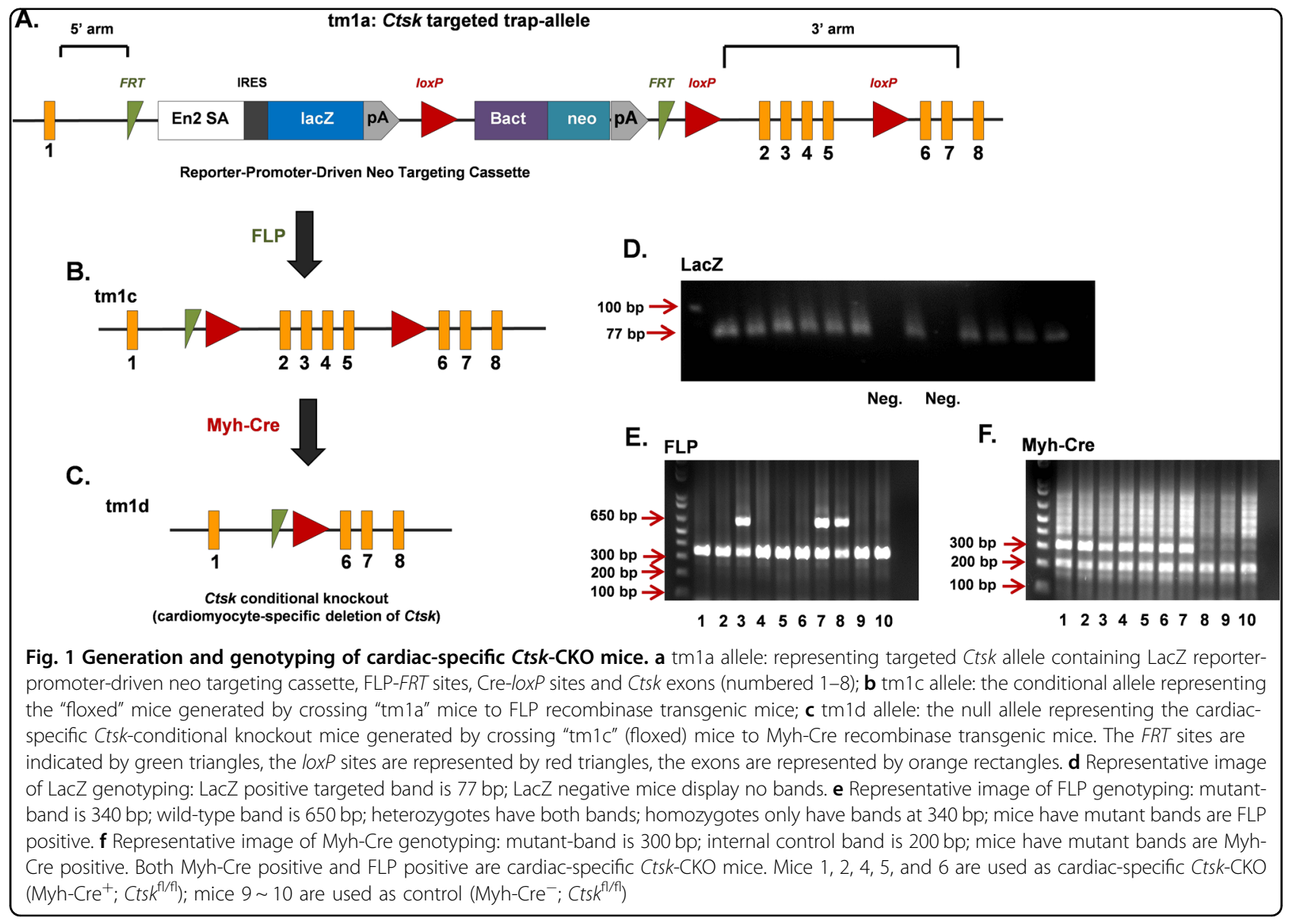




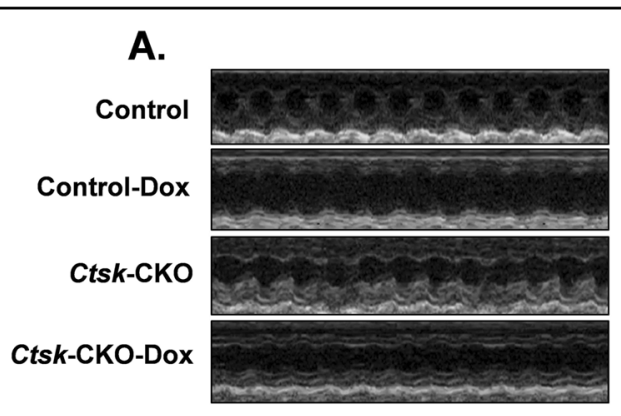

B.

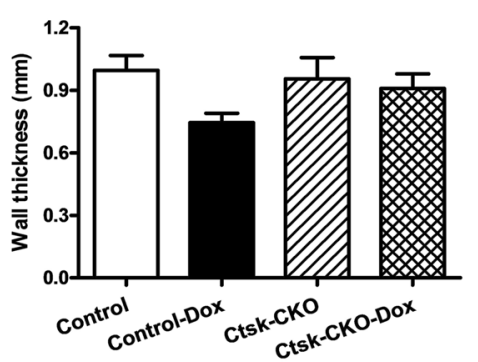

C

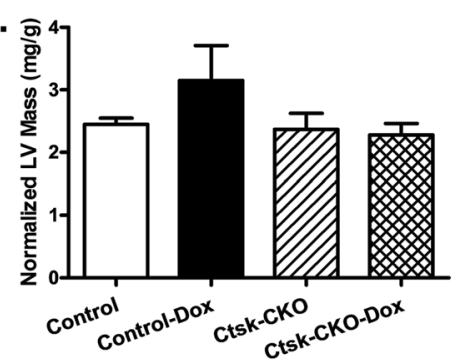

D.

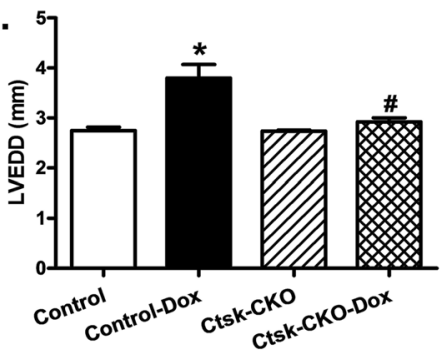

E.

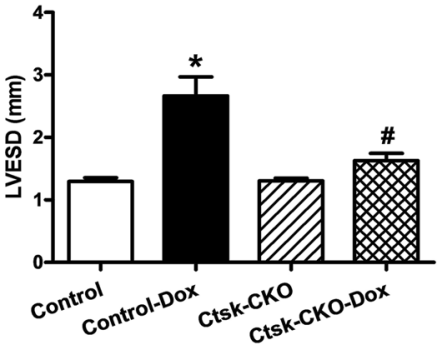

F.

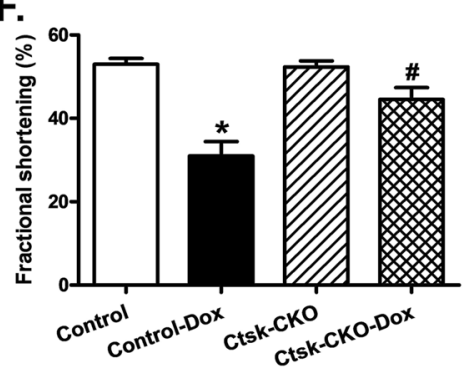

Fig. 2 Echocardiographic properties in control and cardiac-specific Ctsk-CKO mice treated with or without doxorubicin. a Representative echocardiographic images; b Wall thickness; c Left ventricular (LV) mass normalized to body weight; d LV end-diastolic diameter; e LV end-systolic diameter; f Fractional shortening. Mean $\pm \mathrm{SEM}, n=6-8$ mice per group. ${ }^{*} p<0.05$ vs. Control group, $\# p<0.05$ vs. Control-Dox group

significant differences in the levels of Cathepsin K between wild type (WT) and control mice (Supplementary Figure 1E-F).

\section{General biometric and echocardiographic properties}

Doxorubicin treatment resulted in decreased body weight gain in both control and Ctsk-CKO mice, without significant alterations in their heart mass (Supplementary Figure 2). Representative echocardiographic images are shown in Fig. 2a. The wall thickness and normalized LV mass were comparable among control and Ctsk-CKO mice in both vehicle and doxorubicin-treated groups (Fig. 2b, c). However, treatment with doxorubicin significantly increased left ventricular end-diastolic dimension (LVEDD) and left ventricular end-systolic dimension (LVESD) in the control mice but not in the Ctsk-CKO mice (Fig. 2d, e). Furthermore, cardiomyocyte-specific Ctsk deficiency reconciled doxorubicin-induced depressed fractional shortening (Fig. 2f).

\section{Cardiomyocyte contractile function and intracellular $\mathrm{Ca}^{2+}$ properties}

As depicted in Fig. 3a, cardiac-specific Cathepsin K-deficiency prevented the increase in resting cardiomyocyte length induced by doxorubicin. However, cardiomyocytes isolated from control mice challenged with doxorubicin exhibited a significantly reduced peak shortening (PS) and maximal velocity of shortening/ relengthening $( \pm \mathrm{dL} / \mathrm{dt})$, as well as prolonged timeto-90\% relengthening (TR90), all of which were markedly diminished in cardiomyocytes isolated from Ctsk-CKO mice treated with doxorubicin (Fig. 3b-d,f). There was no significant difference in time-to-peak shortening (TPS) in isolated cardiomyocytes between control and Ctsk-CKO mice, with or without doxorubicin treatment (Fig. 3e). In addition, data presented in Fig. 4 reveals a significantly depressed intracellular $\mathrm{Ca}^{2+}$ rise in response to electrical stimulus ( $\Delta \mathrm{FFI})$ (Fig. $4 \mathrm{~b}$ ), reduced intracellular $\mathrm{Ca}^{2+}$ decay rate (single- and 

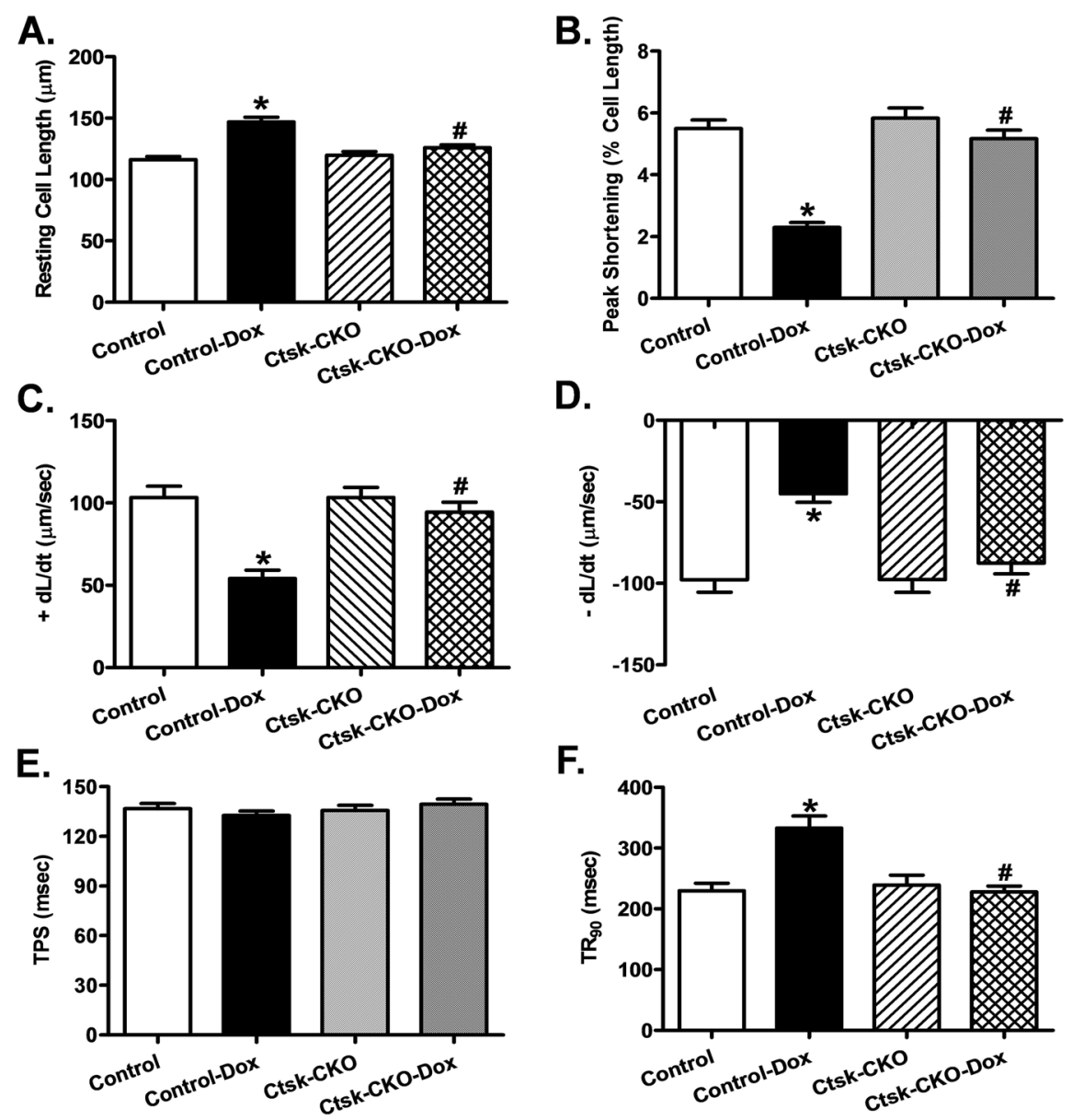

F.

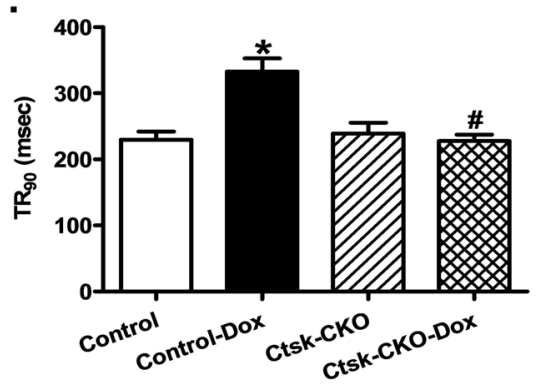

Fig. 3 Cardiomyocyte contractile properties in control and cardiac-specific Ctsk-CKO mice treated with or without doxorubicin. a Resting cell length; $\mathbf{b}$ Peak shortening (PS), normalized to cell length; c Maximal velocity of shortening $(+\mathrm{dL} / \mathrm{dt})$; $\mathbf{d}$ Maximal velocity of relengthening $\left(-\mathrm{dL} / \mathrm{dt}\right.$ ); e Time-to PS (TPS); f Time-to-90\% relengthening (TR90). Mean \pm SEM, $n=88-102$ cells from three to four mice per group. ${ }^{*} p<0.05$ vs. Control group, \#p<0.05 vs. Control-Dox group

Bi-exponential curve fit) (Fig. 4c, d), and an unchanged resting intracellular $\mathrm{Ca}^{2+}$ concentration (Fig. 4a) in cardiomyocytes isolated from doxorubicin-treated control mice compared to those isolated from vehicletreated mice. Cardiac-specific Cathepsin K-deficiency negated doxorubicin-induced prolongation in intracellular $\mathrm{Ca}^{2+}$ decay and depression in $\triangle$ FFI without affecting the baseline FFI. Furthermore, Cathepsin K inhibitor-II reconciled doxorubicin-induced myocyte contractile anomalies including reduced PS and $\pm \mathrm{dL} / \mathrm{dt}$, as well as prolonged $\mathrm{TR}_{90}$ (Supplementary Figure 4 ). Additionally, this effect was blunted by the pretreatment of the cardiomyocytes with apoptosis activator II or the AMPK activator AICAR. On the other hand, inhibition NF-KB using PDTC partially attenuated doxorubicin-triggered abnormal cardiomyocyte contractile function. TPS was not significantly affected by any of the drug treatment.

\section{Cardiomyocyte cross-sectional area, fibrosis, and myofibrillar disruption}

To evaluate the impact of Ctsk-CKO on myocardial histology in doxorubicin-treated mice, cardiomyocyte cross-sectional area, myofibrillar disruption and cardiac fibrosis were examined (Fig. 5). Masson trichrome staining revealed the presence of cardiac fibrosis in the hearts of both control and Ctsk-CKO mice treated with doxorubicin; whereas, myofibrillar disruption was seen only in doxorubicin-treated control mice (Fig. 5a). Hematoxylin and eosin staining and lectin staining of the histologic sections showed decreased cardiomyocyte cross-sectional area, cytoplasmic vacuolization and myocytolysis (myofibrillar disruption) following doxorubicin treatment. In contrast, cardiomyocytes from Ctsk-CKO mice subjected to doxorubicin did not exhibit these anomalies (Fig. 5b-e). Semi-quantitative analysis of myocardial vacuolization and myofibrillar degeneration is provided in 
A.
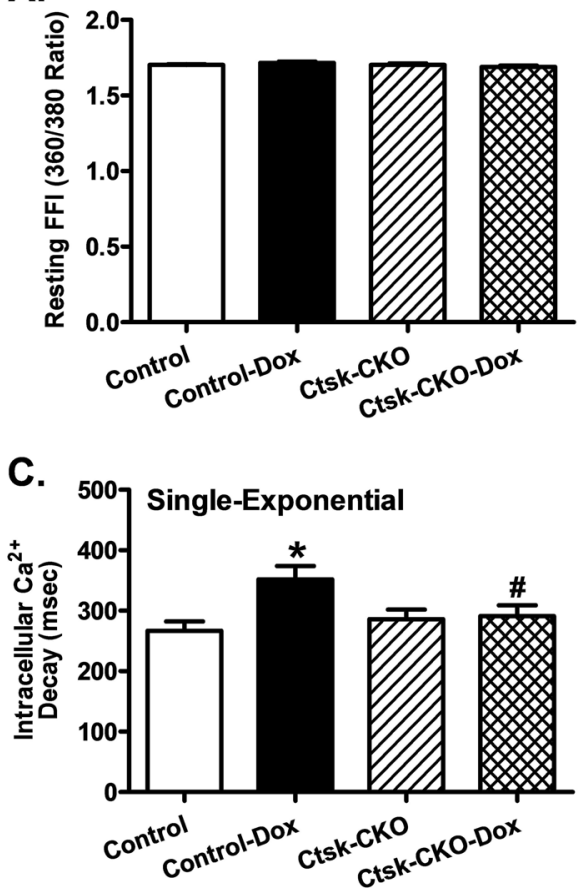

B.
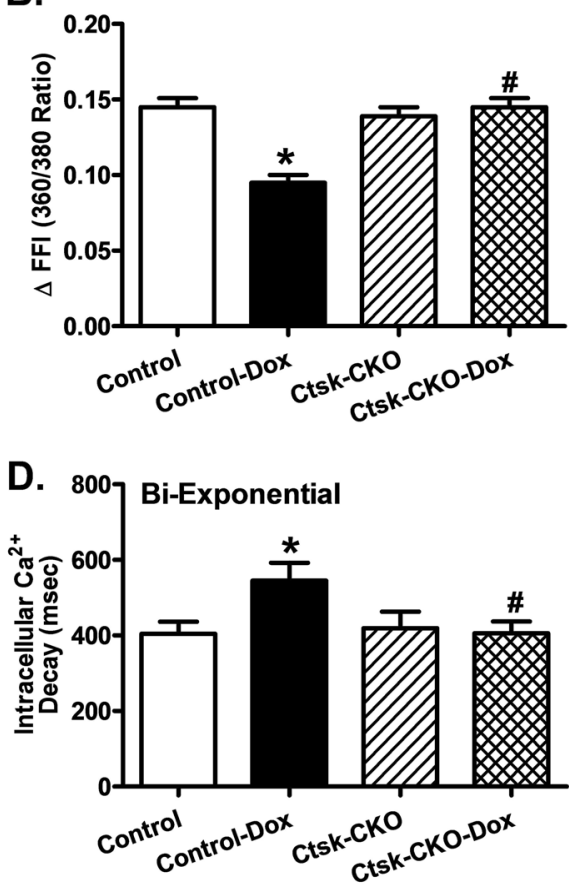

Fig. 4 Cardiomyocyte intracellular $\mathrm{Ca}^{2+}$ handling properties in control and cardiac-specific Ctsk-CKO mice treated with or without doxorubicin. a Resting fura-2 fluorescence intensity (FFI); b Electrically stimulated rise in FFI ( $\triangle \mathrm{FFI}$ ); c Intracellular $\mathrm{Ca}^{2+}$ decay rate (single exponential); d Intracellular $\mathrm{Ca}^{2+}$ decay rate (bi-exponential). Mean $\pm \mathrm{SEM}, n=80-96$ cells from three to four mice per group. ${ }^{*} p<0.05$ vs. Control group, \#p $<0.05$ vs. Control-Dox group

Fig. 5f. Next, sarcomeric cytoskeletal proteins, including desmin and $\alpha$-actinin, as well as Cathepsin $K$ in the myocardium were determined by western blot (Fig. 6). Doxorubicin challenge upregulated the expression of Cathepsin $\mathrm{K}$ protein while downregulating desmin and sarcomeric $\alpha$-actinin. Additionally, doxorubicin-induced attenuation of desmin and sarcomeric $\alpha$-actinin were negated in Ctsk-CKO mice.

\section{Cardiac apoptosis, inflammation, metabolic and survival signaling}

Because doxorubicin has been shown to mediate its deleterious effects via inflammation and cardiac apoptosis, as well as the disturbance of cardiac energy metabolism, we next determined the effect of Ctsk-CKO on cardiac apoptosis, inflammation, metabolic, and survival signaling in the myocardium. To this end, we measured the phosphorylation levels of metabolic regulators AKT and AMPK $\alpha$, pro-apoptotic markers BAX and cleaved caspase-3, anti-apoptotic marker Bcl-2, as well as mediators

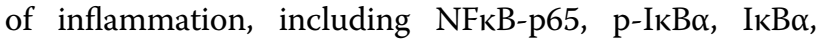
IL-1 $\beta$, IL-6, and TNF- $\alpha$ using western blot analysis. Moreover, glycolytic and oxidative flux was measured by determining the formation of cardiac lactate from pyruvate. Our data revealed that doxorubicin treatment increased levels of $\mathrm{p}-\mathrm{AMPK} \alpha, \mathrm{BAX}$, cleaved caspase-3, NFкB-p65, p-IкB $\alpha$, and IL-6 and dampened p-AKT and $\mathrm{Bcl}-2$, all of which were mitigated in the Ctsk-CKO mice. There were no significant changes in protein expression of I $\mathrm{KB} \alpha$, IL-1 $\beta$, and TNF- $\alpha$ with either the knockout or doxorubicin challenge (Fig. 7). Cardiac lactate production was elevated in doxorubicin-treated control mice, which was reversed in Ctsk-CKO mice (Supplementary Figure 3). To further ascertain changes in apoptosis, we performed TUNEL staining on our tissue sections. The TUNEL-positive nuclei visualized in fluorescein green as a percentage of all nuclei stained with DAPI (blue) were significantly higher in the myocardium from doxorubicin-treated control mice. In contrast, we observed fewer TUNEL-positive nuclei in the knockout mice subjected to doxorubicin. Knockout of Ctsk by itself did not affect the TUNEL-positive nuclei in the absence of doxorubicin treatment (Fig. 8).

\section{Discussion}

Our study demonstrated that cardiomyocyte-specific knockout of Cathepsin $\mathrm{K}$ mitigates doxorubicin-induced cardiac structural and functional defects, suggesting a direct involvement of Cathepsin $\mathrm{K}$ in cardiotoxicity mediated by doxorubicin. Here we provide the evidence 
A.
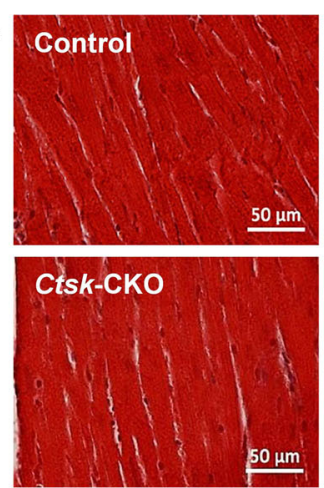

B.

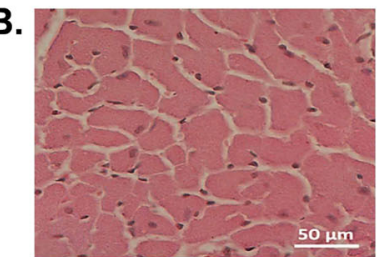

C.

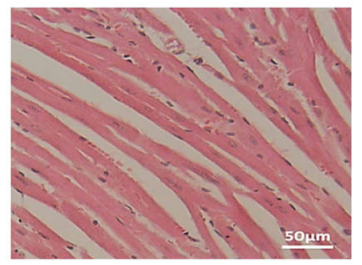

D.

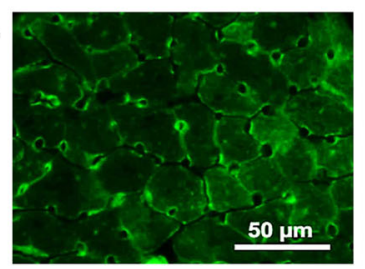

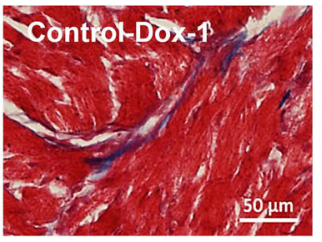

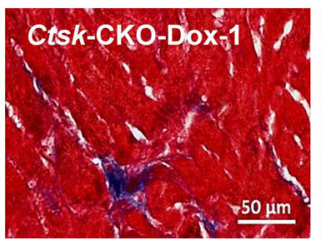

Control-Dox
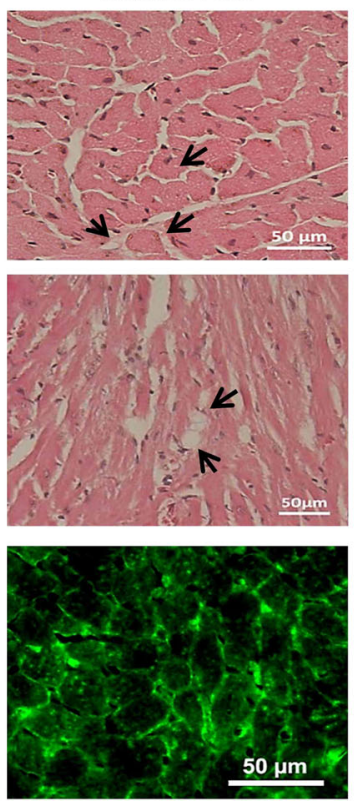
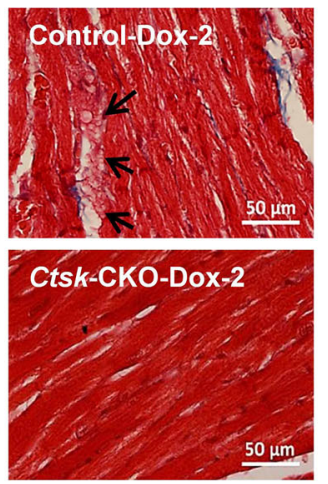

Ctsk-CKO
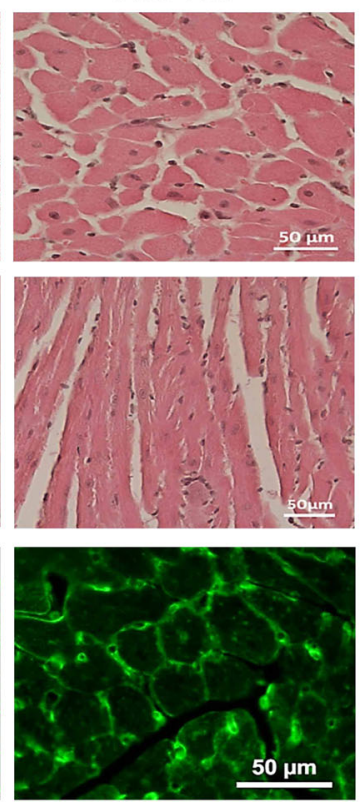

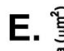

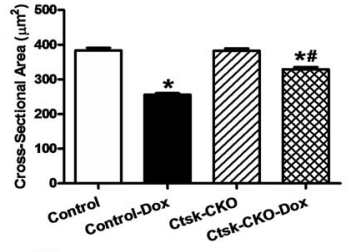

F.

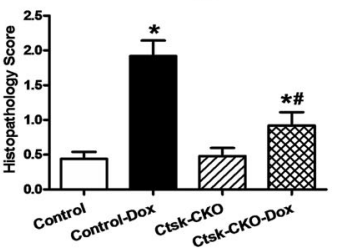

Ctsk-CKO-Dox
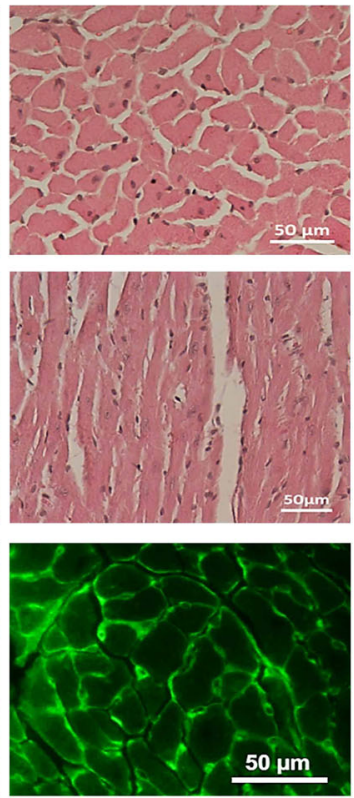

Fig. 5 Histological analyses in hearts from control and cardiac-specific Ctsk-CKO mice treated with or without doxorubicin. a Representative images of Masson trichrome staining for fibrosis $(\times 400$; scale bar $=50 \mu \mathrm{m})$; b Representative H\&E staining micrographs of transverse sections of left ventricular myocardium $(\times 400$; scale bar $=50 \mu \mathrm{m})$; c Representative H\&E staining micrographs of longitudinal sections of left ventricular myocardium $(\times 200$; scale bar $=50 \mu \mathrm{m})$; $\mathbf{d}$ Representative Lectin staining of transverse sections of left ventricular myocardium $(\times 400$; scale bar $=50 \mu \mathrm{m})$; e Quantitative cardiomyocyte cross-sectional (transverse) area using measurements of 230 cardiomyocytes from three mice per group; f Semi-quantitative vacuolization and myofibrillar degeneration of the cardiomyocytes by scoring scales from 25 slides per group. Mean \pm SEM, ${ }^{*} p<0.05$ vs. Control group, $\# p<0.05$ vs. Control-Dox group. Arrow: cytoplasmic vacuolization and myofibrillar degeneration

that cardiomyocyte-specific deletion of Cathepsin K attenuated or ablated doxorubicin-induced aberrant cardiac structure and function, abnormal cardiomyocyte morphology, myofibrillar degeneration, impaired energy metabolism, inflammatory response, and apoptosis. These findings not only support our previous studies that global knockout of Cathepsin K reconciled streptozotocin-, high-fat diet-, and pressure overload-induced cardiac dysfunction $^{8,9,11}$, but extend these findings to show that the deleterious effects of Cathepsin $\mathrm{K}$ in these models are, at least in part, attributable to its cardiac-specific effects. The protective effects of Cathepsin $\mathrm{K}$ ablation were associated with alterations in multiple signaling pathways, including restoration of phosphorylation of AMPK, reduction in pro-apoptotic proteins BAX and cleavedcaspase-3, blunted NF- $\mathrm{KB}$ signaling, as well as recovery of phosphorylation of AKT.

Previous studies have reported that Cathepsin K levels were elevated in the hearts of human subjects and experimental animals with dilated cardiomyopathy, hypertrophic cardiomyopathy, and heart failure ${ }^{5,8,11,16}$. While our current study confirms the upregulation of Cathepsin $\mathrm{K}$ expression in myopathic heart following doxorubicin treatment, it also supports the notion that 

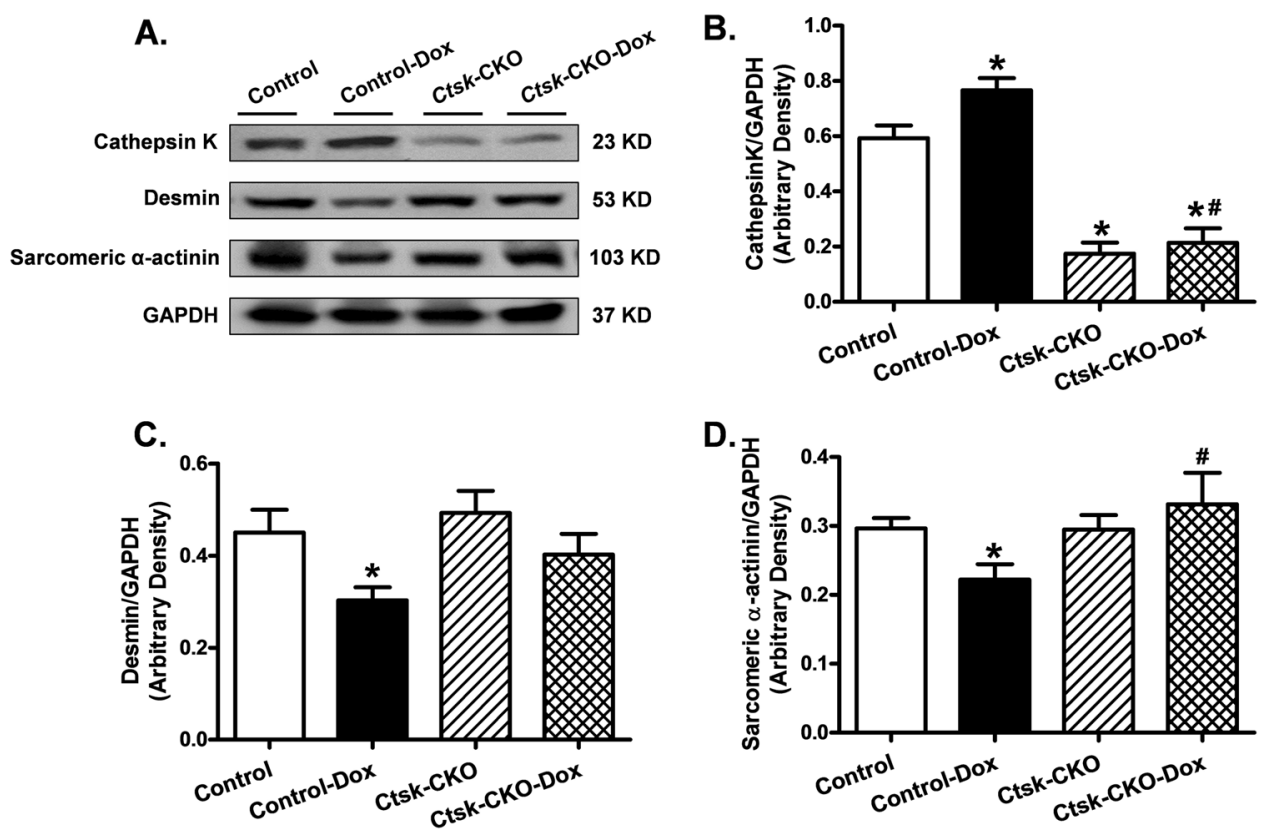

Fig. 6 Western blot analysis exhibiting levels of Cathepsin $\mathrm{K}$ and myofibrillar protein markers in myocardium from control and cardiacspecific Ctsk-CKO mice treated with or without doxorubicin. a Representative gel blots depicting expressions of Cathepsin K, desmin, sarcomeric a-actinin, and GAPDH (loading control); b Cathepsin K/GAPDH; c Desmin/GAPDH; d Sarcomeric a-actinin/GAPDH. Mean \pm SEM, $n=6-8$ mice per group, ${ }^{*} p<0.05$ vs. Control group, \#p $<0.05$ vs. Control-Dox group

Cathepsin $\mathrm{K}$ upregulation may be a cause rather than consequence of the disease. Consistent with previous studies, doxorubicin administrated resulted in enlargement of left ventricular chamber assessed as increased LVESD and LVEDD, as well as reduced fractional shortening in control hearts, reminiscent of cardiac geometric changes associated with dilated cardiomyopathy and cardiac remolding ${ }^{12,}{ }^{17-19}$. These changes were dramatically attenuated in Ctsk-CKO mice, indicating that the absence of Cathepsin $\mathrm{K}$ in the myocardium may protect against cardiac remodeling and dysfunction. Moreover, data from this study showed that Ctsk-CKO reconciled doxorubicin-induced cardiomyocyte contractile dysfunction and disturbed intracellular $\mathrm{Ca}^{2+}$ homeostasis manifested as suppressed peak shortening and maximal velocity of shortening/ relengthening, prolonged time-to- $90 \%$ relengthening, as well as depressed electrically stimulated rise and clearance in intracellular $\mathrm{Ca}^{2+}$, which is consistent with our previous work using global Cathepsin $\mathrm{K}$ knockout mice ${ }^{8 \text {, }}$ ${ }^{11}$. The length of single cardiomyocyte was significantly increased; whereas, the cross-sectional area of the cardiomyocytes was remarkably decreased by doxorubicin treatment. Furthermore, doxorubicin caused a severe cardiac cytoplasmic vacuolization and myofibrillar disruption with a loss-of-desmin and sarcomeric $\alpha$-actinin expression in control mice. All these effects were markedly ameliorated by cardiac-specific deletion of Cathepsin K, suggesting a critical role of cardiac Cathepsin $\mathrm{K}$ in myofibrillar remodeling and cardiomyocyte injury, contributing to the doxorubicin-induced whole heart structure and functional changes that are similar to dilated cardiomyopathy.

The impaired myofibrils and myocyte vacuolization as typical features of doxorubicin cardiotoxicity have been observed in several previous studies ${ }^{12,}{ }^{19-22}$, and the mutation or deletion of cytoskeletal proteins, such as $\alpha$ actinin and desmin have been shown to contribute to dilated cardiomyopathy ${ }^{23}, 24$. Desmin, as a major intermediate filament cytoskeleton, makes the organization of myofibrils and maintains the structural and mechanical integrity of cardiomyocytes ${ }^{25}$. Loss-of-desmin was detected at the end stage of heart failure ${ }^{26}$. Mice lacking desmin were found to develop dilated cardiomyopathy, smooth muscle, and skeletal muscle defects ${ }^{25}$, 27, 28. Similarly, $\alpha$-actinin localized at the Z-disk is a cytoskeletal actinbinding protein, which also plays important structural and regulatory roles in cytoskeletal arrangement and muscle contraction ${ }^{29}, 30$. Defects in sarcomeric $\alpha$-actinin (ACTN2) can cause both hypertrophic and dilated cardiomyopathy $^{31,32}$. These cytoskeletal defects may be associated with apoptosis, and act as compensatory responses to apoptotic activation, leading to cardiomyocyte contractile dysfunction before cell death ${ }^{33-35}$. Other 

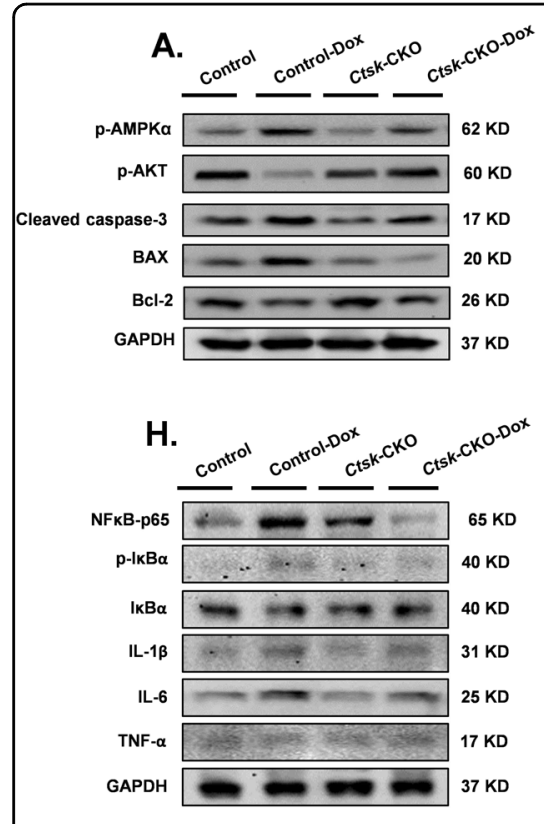

L.

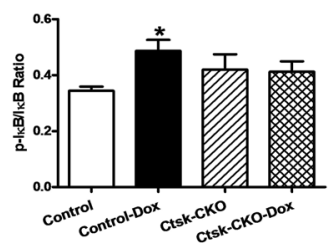

B.
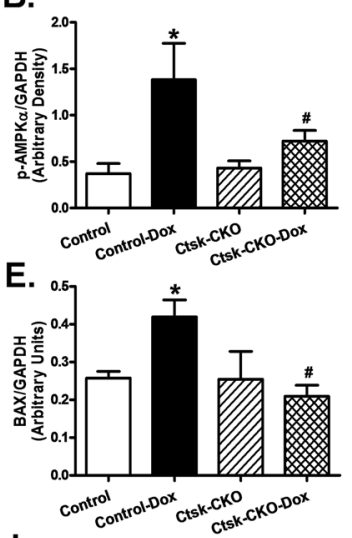

I.

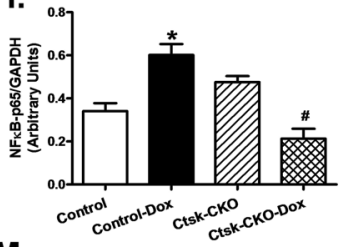

M.

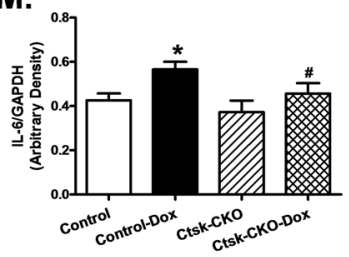

C.

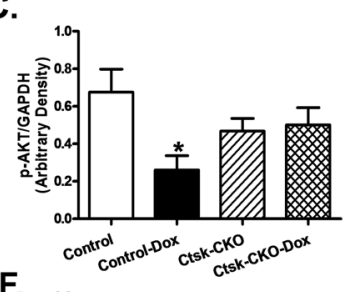

F.

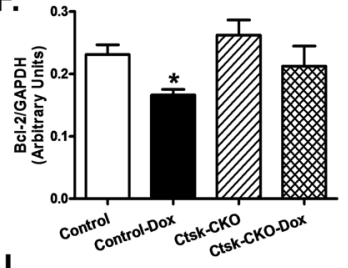

J.

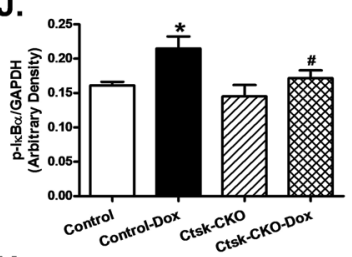

N.

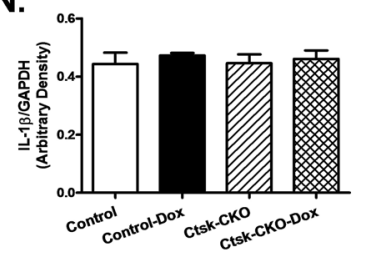

D.

G.

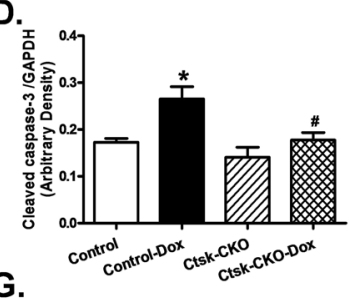

K.
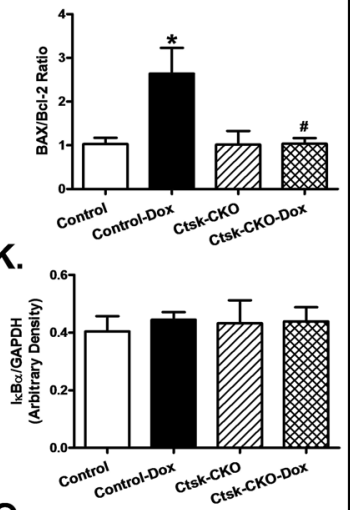

0 .

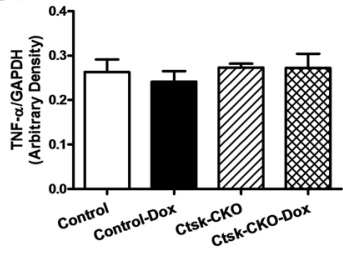

Fig. 7 Western blot analysis exhibiting levels of metabolic and survival signaling, apoptotic markers, and inflammatory markers in myocardium from control and cardiac-specific Ctsk-CKO mice treated with or without doxorubicin. a Representative gel blots depicting expressions of p-AMPKa, p-AKT, cleaved caspase-3, BAX, Bcl-2, and GAPDH (loading control); b p-AMPKa/GAPDH; c p-AKT/GAPDH; d Cleavedcaspase-3/GAPDH; e BAX/GAPDH; $\mathbf{f}$ BCl-2/GAPDH; $\mathbf{g}$ BAX/BCl-2 ratio; $\mathbf{h}$ Representative gel blots depicting expressions of NFKB-p65, phosphorylated

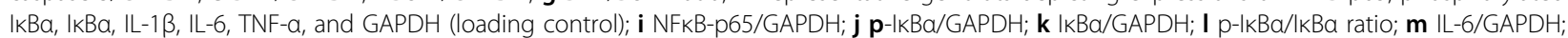

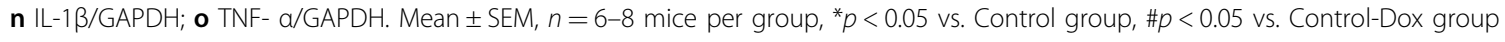

studies also have shown that caspase-3 is able to cleave $\alpha$ actin and desmin, resulting in an impaired $\mathrm{Ca}^{2+}$-activated force and myofibrillar ATPase activity, and promoting apoptosis $^{35,36}$. Our present study revealed a significant enhancement of apoptosis in the heart following doxorubicin treatment as evidenced by an increase in the protein levels of cleaved-caspase-3 and BAX, and decreased Bcl-2, accompanied with reduced desmin and sarcomeric $\alpha$-actinin, as well as upregulation of Cathepsin $K$, all of which were attenuated in cardiac Cathepsin $\mathrm{K}$ knockout mice. These results indicate that the deletion of Cathepsin $\mathrm{K}$ in the cardiomyocyte prevents doxorubicin-activated apoptosis and subsequent myofibrillar degeneration and contractile dysfunction. Other proteases such as calpain and Cathepsin B have been reported to cleave desmin, which may also be a substrate for Cathepsin $\mathrm{K}^{37}$. Interestingly, the absence of cardiac Cathepsin $\mathrm{K}$ had no effect on doxorubicin-induced myocardial fibrosis, suggesting that the protective role of Ctsk-CKO against doxorubicin toxicity may be independent of extracellular matrix remodeling.

In this study, we also demonstrated that cardiomyocytespecific Cathepsin K knockout alleviated doxorubicininduced AMPK and NF- $\mathrm{KB}$ activation in cardiac tissue, and restored phosphorylation of AKT, which are blunted following doxorubicin treatment. Accumulating evidence indicates a vital role of perturbation of energy metabolism and mitochondrial dysfunction in cardiac pathological responses to doxorubicin administration ${ }^{38,} 39$. The energy sensor AMP-activated protein kinase (AMPK) is extremely sensitive to cellular AMP and ATP levels and is activated by elevated AMP/ATP ratio ${ }^{40}$. The increased phosphorylation of AMPK by doxorubicin suggests disruption of mitochondria, resulting in a significant depletion of cellular ATP, which may increase AMP/ATP ratio $^{41}$. In contrast, previous studies have suggested that AMPK signaling is inhibited in the heart following doxorubicin treatment in a Langendorff rat-model ${ }^{42}$. The 


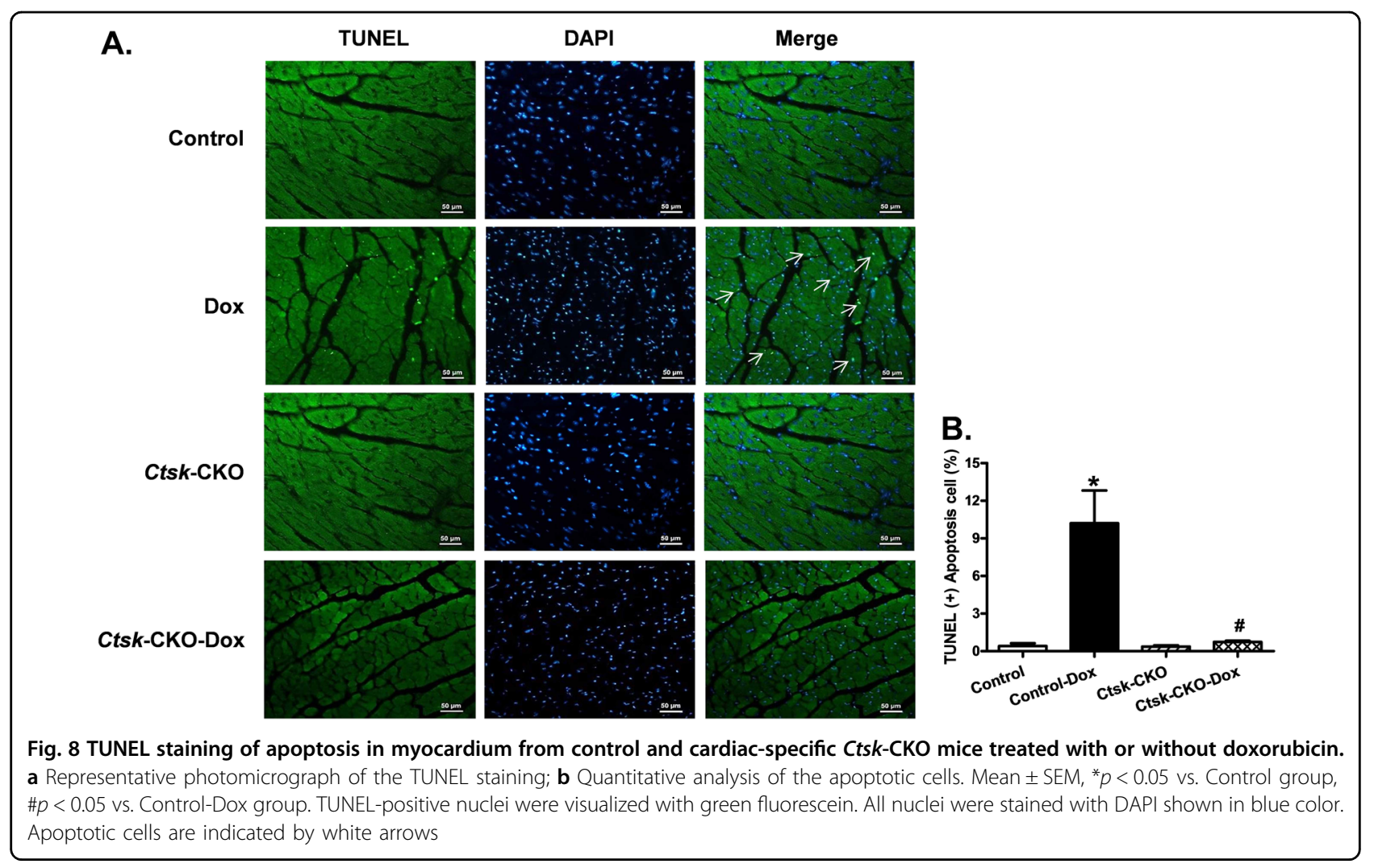

difference in results between that study and our present study may be attributed to the different models and/or differences in the dosage and regimen of doxorubicin administration. Additionally, doxorubicin can accelerate membrane lipid peroxidation, leading to the production of cytotoxic end-products, and the disruption of unsaturated fatty acyl chains ${ }^{39}$. These effects may be associated with the activation of AMPK, which functions to regulate the cellular lipid and glucose metabolism. To further discern the potential involvement of Cathepsin $\mathrm{K}$ in glycolytic changes we examined cardiac lactate levels. In organisms, the lactate is the end product of glycolysis. Under stressed condition or with circulatory dysfunction, more lactate is generated by the conversion of pyruvate, which catalyzed by the enzyme lactate dehydrogenase. Enhanced circulating and myocardial levels of lactate dehydrogenase have been recognized as a biomarker for cardiomyopathy and heart failure and is closely associated with cardiac dysfunction ${ }^{43-46}$. Our results revealed that doxorubicin treatment resulted in elevated lactate levels in the heart, which may suggest a decreased oxidative phosphorylation, leading to reductions in ATP production. The cardiac energy stress caused by reduced ATP after doxorubicin treatment may be compensated with higher AMPK to promote both lipid and glucose metabolism. Recent studies also suggest that AMPK activation can promote apoptosis ${ }^{47}$. Additionally, activation of $\mathrm{NF}-\mathrm{KB}$ has been considered as an alternative mechanism leading to doxorubicin-induced apoptosis in cardiomyocytes $^{48}$. The p65 subunit of NF- $\mathrm{kB}$ can cause metabolic disturbance and inflammation by PGC-1 $\alpha^{49}$. Additionally, we found a significant increase in the pro-inflammatory cytokine IL-6 in the myocardium, which is reported to be associated with the development of heart failure ${ }^{50}$. In contrast, we did not observe any changes in the levels of IL- $1 \beta$ and TNF- $\alpha$. A plausible explanation for this is that while IL-6 is induced after transient stimulation with doxorubicin, TNF- $\alpha$ was found to be induced at higher doses with repeated injections ${ }^{51,52}$. Furthermore, decreased $\mathrm{p}$-AKT induced by doxorubicin indicates a reduced survival signal in the cardiomyocytes, and is consistent with elevated levels of apoptosis ${ }^{53}$. Most importantly, these changes were not seen in Cathepsin K conditional knockout mice, confirming the benefit of targeting Cathepsin $\mathrm{K}$ to improve cardiovascular and metabolic homeostasis with anti-inflammatory and antiapoptotic response.

To further understand the potential role of the activation of AMPK and NF- $\mathrm{BB}$, as well as apoptosis in the protective effects of Cathepsin $\mathrm{K}$ ablation in doxorubicininduced cardiotoxicity, we examined the effect of an AMPK activator AICAR, NF- $\mathrm{kB}$ inhibitor PDTC and 
apoptosis activator II on cardiomyocyte contractile function in the presence or absence of Cathepsin $\mathrm{K}$ inhibitor and doxorubicin treatment. Cathepsin $\mathrm{K}$ inhibitor-II restored doxorubicin-induced myocyte contractile anomalies, which was blunted by the pre-treatment of apoptosis activator II and AICAR, suggesting a role of AMPK and apoptotic signaling in the cardioprotection accorded by Cathepsin K knockout. PDTC on the other hand, partially attenuated doxorubicin-induced abnormal cardiomyocyte contractility, indicating that NF- $\mathrm{kB}$ activation may not be the primary mechanism involved. Further studies using knockout and overexpression of specific proteins are necessary to ascertain the role of these pathways that the cardioprotection offered by ablation of Cathepsin K.

In summary, our present study demonstrates, for the first time, that cardiac-specific deletion of Cathepsin $\mathrm{K}$ rescues mice from doxorubicin-induced cardiac structural and functional anomalies, which may be mediated by reconciling doxorubicin-induced imbalance of cardiac energy metabolism, NF- $\mathrm{kB}$ activation, and elevated apoptosis. These observations are particularly significant given the fact that Cathepsin $\mathrm{K}$ is upregulated in the failing heart. Inhibition of Cathepsin $\mathrm{K}$ could represent a therapeutic strategy in the management of cardiac dysfunction.

\section{Methods and materials}

Generation of cardiac Ctsk-CKO mice and treatments

To generate cardiac Cathepsin K knockout mice (CtskCKO mice), three JM8 embryonic stem (ES) cell clones (HEPD0727 clones C11, F11, and H09), with conditional potential targeting exon 2-5 of Ctsk (Fig. 1a) were obtained from the European Mouse Mutant Cell Repository (EuMMCR). Male ES cells (C57BL/6N genetic background) were injected into C57BL/6 blastocysts at the University of Washington Transgenic Resources Program. The chimeras and the resulting germline offspring are from the $\mathrm{C} 11$ clone. The targeted Ctsk allele contains a reporter-promoter LacZ, FLP-FRT and CreloxP sites (Fig. 1a). Pups were genotyped for the presence of LacZ, using a LacZ TaqMan primer/probe marker set purchased from Applied Biosystems/Life Technologies and the probe Mr03987581_mr (amplicon length $77 \mathrm{bp}$ ) (Fig. 1d). These mice were crossed with mice expressing flippase (FLP) recombinase (B6.129S4-Gt(ROSA) 26Sor $<\operatorname{tm} 2\left(\mathrm{FLP}^{*}\right)$ Sor $>/ \mathrm{J}$ (The Jackson Laboratory) ${ }^{54}$, resulting in offspring in which the LacZ reporter and neo cassette had been deleted (Fig. 1b). FLP positive LacZ negative mice were then crossed with those expressing Cre recombinase under control of the cardiac-specific murine alpha myosin-heavy chain (myh6) promoter (B6N.FVB(B6)-Tg(Myh6-cre)2182Mds/J; The Jackson

Table 1 Primer sequence for genotyping and RT-PCR

\begin{tabular}{|c|c|c|c|}
\hline \multicolumn{3}{|c|}{ Protocol primers for FLP genotyping } & \multirow{2}{*}{$\begin{array}{l}\text { Reference/note } \\
\text { Designed by the Jackson Lab }\end{array}$} \\
\hline Primer & Sequence $5^{\prime}-->3^{\prime}$ & Primer type & \\
\hline olMR8545 & 5'-AAAGTCGCTCTGAGTTGTTAT-3' & WT and mutant forward & \\
\hline OlMR8546 & 5'-GGAGCGGGAGAAATGGATATG-3' & WT reverse & \\
\hline OlMR8502 & 5'-GCGAAGAGTTTGTCCTCAACC-3' & Mutant reverse & \\
\hline \multicolumn{4}{|c|}{ Protocol primers for Myh-Cre genotyping } \\
\hline Primer & Sequence $5^{\prime}-->3^{\prime}$ & Primer type & Designed by the Jackson Lab \\
\hline OlMR8744 & 5'-CAAATGTTGCTTGTCTGGTG-3' & Internal positive control forward & \\
\hline olMR8745 & 5'-GTCAGTCGAGTGCACAGTTT-3' & Internal positive control reverse & \\
\hline 9543 & 5'-ATGACAGACAGATCCCTCCTATC-3' & Transgene forward & \\
\hline 9544 & 5'-CTCATCACTCGTTGCATCATCGA-3 & Transgene reverse & \\
\hline \multicolumn{4}{|c|}{ RT-PCR primers for mouse Cathepsin $\mathrm{K}$ and $18 \mathrm{~S}$} \\
\hline Primer & Sequence $5^{\prime}-->3^{\prime}$ & Primer type & Designed on IDT \\
\hline Ctsk-exon 2 & 5'-TCAAGTTTCTGCTGCTACC-3' & Forward & \\
\hline Ctsk-exon 2 & 5'-GCTTCTGGTGAGTCTTCTTC-3' & Reverse & \\
\hline Ctsk & 5'-GGGCCAGGATGAAAGTTGTA-3' & Forward & 8 \\
\hline Ctsk & 5'-CACTGCTCTCTTCAGGGCTT-3' & Reverse & \\
\hline Rn18s & 5'-AGTGACAAGAAATAACAATACAGG-3' & Forward & \\
\hline Rn18s & 5'-CCTGCTTTAAGCACTCTAATTTTC-3' & Reverse & \\
\hline
\end{tabular}


Laboratory; C57BL/6 background), as shown in Fig. 1c to generate both control (Myh-Cre ${ }^{-} ;$Ctsk $^{\mathrm{fl} / \mathrm{fl}}$ ) and cardiomyocyte-specific Ctsk-CKO (Myh-Cre ${ }^{+}$; Ctsk $^{\text {fl/fl }}$ ) littermate mice. Control and Ctsk-CKO, as well as Ctsk floxed mice were genotyped by PCR using FLP and MyhCre primers (Fig. 1e,f). The sequences of the primers are shown in Table 1. Both FLP and Myh-Cre primers were designed by the Jackson Lab. The experimental procedure was approved by the Institutional Animal Use and Care Committees of the University of Wyoming, Laramie, WY and the University of Washington, Seattle, WA). Fourmonth-old control and Ctsk-CKO mice (both male and female) weighing $25 \sim 28 \mathrm{~g}$ were randomly assigned to injections of doxorubicin $(10 \mathrm{mg} / \mathrm{kg}$, i.p. at 3-day intervals, $20 \mathrm{mg} / \mathrm{kg}$ cumulative) or vehicle ${ }^{18}$. One week after the first injection, body and tissue weight, echocardiographic properties, cardiomyocyte contractile properties, and calcium handling were evaluated. Cardiac structure was assessed by histomorphology. Myofibrillar protein markers, NF- $\mathrm{kB}$ signaling, and apoptosis were determined by western blot.

\section{Echocardiographic assessment}

Cardiac geometry and function were evaluated in anesthetized (isoflurane $1.2 \%$ administered with a calibrated vaporizer and an inhalant) mice using a twodimensional (2D) guided M-mode echocardiography (Phillips Sonos 5500) equipped with a $15-6 \mathrm{MHz}$ linear transducer (Phillips Medical Systems, Andover, MD, USA). Adequate depth of anesthesia was monitored using toe reflex. The heart was imaged in the 2D-mode in the parasternal long-axis view with a depth of $2 \mathrm{~cm}$. The M-mode cursor was positioned perpendicular to interventricular septum and posterior wall of left-ventricle (LV) at the level of papillary muscles from the 2D-mode. Diastolic wall thickness, end-diastolic dimension (EDD) and end-systolic dimension (ESD) were measured. All measurements were done from leading edge to leading edge in accordance with the Guidelines of the American Society of Echocardiography. LV mass was calculated as 0.8(1.04[([LVEDD + IVSd + PWd]3-LVEDD3)] +0.6 . LV fractional shortening was calculated as [(EDD-ESD)/ EDD] $\times 100$. Normalized LV mass was calculated as LV mass (mg)/body weight $(\mathrm{g})^{55}$.

\section{Isolation of cardiomyocytes and the treatment}

Cardiomyocytes were isolated from wild type (WT), control and conditional knockout mice were treated with doxorubicin or vehicle as described previously ${ }^{56}$. After ketamine/xylazine sedation, hearts were removed and perfused with $\mathrm{Ca}^{2+}$-free HEPES-buffered Tyrode's solution containing (in $\mathrm{mM}$ ): $\mathrm{NaCl} 120, \mathrm{KCl} 15, \mathrm{KH}_{2} \mathrm{PO}_{4} 0.6$, $\mathrm{Na}_{2} \mathrm{HPO}_{4}$ 0.6, $\mathrm{NaHCO}_{3}$ 4.6, $\mathrm{MgSO}_{4}$ 1.2, HEPES 10, taurine 30 , glucose 10 , butanedione monoxime 10 at $\mathrm{pH}$
7.4 , and gassed with $95 \%$ oxygen and $5 \%$ carbon dioxide. Hearts were digested with $1 \mathrm{mg}$ Liberase $\mathrm{TH}$ (Roche Diagnostics, Indianapolis, IN, USA) in $20 \mathrm{ml}$ perfusion buffer for 10-15 min to collect the cells. Only rod-shaped myocytes with clear edges were selected for the study. To assess the role of AMPK and apoptosis in Cathepsin K-regulated cardiomyocyte contractile response following doxorubicin exposure, cardiomyocytes from adult WT mice were pre-treated with AMPK activator AICAR $(500 \mu \mathrm{M})$ or apoptosis activator II (AAII, $10 \mu \mathrm{M}) 30 \mathrm{~min}$ prior to the treatment of Cathepsin K inhibitor-II (CatK I, $1 \mu \mathrm{M}$ ) and doxorubicin (Dox, $1 \mu \mathrm{M}, 30 \mathrm{~min})^{57-59}$. To evaluate the role of NF- $\mathrm{kB}$ in response to doxorubicin, cardiomyocytes from WT mice were incubated with NF$\kappa B$-specific inhibitor PDTC $(100 \mu \mathrm{M}, 30 \mathrm{~min})$ along with doxorubicin challenge $(1 \mu \mathrm{M})^{60,61}$.

\section{Cell shortening/relengthening}

Mechanical properties of cardiomyocytes were evaluated using a SoftEdge Myocam $^{\circledast}$ system (IonOptix Corporation, Milton, MA). Cardiomyocytes were placed in a chamber mounted on the stage of an inverted microscope (Olympus IX-70) and superfused with a HEPES buffer containing $1 \mathrm{mM} \mathrm{CaCl} 2$. Myocytes were field stimulated with suprathreshold voltage at a $0.5 \mathrm{~Hz}$ frequency, using a pair of platinum wires placed on opposite sides of the chamber connected to an FHC stimulator (Brunswick, NE). IonOptix SoftEdge software was utilized to capture cell shortening and relengthening changes. Cell shortening and relengthening were assessed using the following indices: resting cell length, peak shortening (PS), time-to-PS (TPS), time-to-90\% relengthening (TR90), and maximal velocity of shortening/relengthening $( \pm \mathrm{dL} / \mathrm{dt})^{56}$.

\section{Intracellular $\mathrm{Ca}^{2+}$ transient analysis}

Cardiomyocytes were loaded with fura-2/AM $(0.5 \mu \mathrm{M})$ for $15 \mathrm{~min}$, and fluorescence intensity was recorded with a dual-excitation fluorescence photomultiplier system (IonOptix). Cardiomyocytes were placed onto an Olympus IX-70 inverted microscope and were imaged through a Fluor $\times 40$ oil objective. Cells were exposed to light excited by a $75 \mathrm{~W}$ lamp and passed through either a 360 or a $380 \mathrm{~nm}$ filter, while being stimulated to contract at a frequency of $0.5 \mathrm{~Hz}$. Fluorescence emissions were detected between 480 and $520 \mathrm{~nm}$ and the qualitative change in fura-2 fluorescence intensity (FFI) was inferred from the FFI ratio at the two wavelengths $(360 / 380 \mathrm{~nm})$. Fluorescence decay time (single exponential) was calculated as an indicator of intracellular $\mathrm{Ca}^{2+}$ clearance ${ }^{56}$.

\section{Myocardial histological analysis}

Following anesthesia, hearts were arrested in diastole with saturated $\mathrm{KCl}$, excised, segmented and immediately 
fixed in $10 \%$ neutral-buffered formalin at room temperature for $24 \mathrm{~h}$. The specimens were then dehydrated through a series of graded alcohols, cleared in xylenes and embedded in paraffin. The serial sections were cut at $7 \mu \mathrm{m}$ and stained with hematoxylin and eosin $(\mathrm{H} \& \mathrm{E})^{62}$. Another set of sections were stained with $0.1 \mathrm{mg} / \mathrm{ml}$ Lectin-FITC conjugate (Sigma-Aldrich, L-4895) for $2 \mathrm{~h}$ at room temperature in the dark ${ }^{63}$. Slides were then washed with PBS and mounted with Fluoromount-G mounting media (Southern Biotech, Inc., Birmingham, AL).Cardiomyocyte cross-sectional area was measured and quantified from 130 random cardiomyocytes from H\&E stained slides and 100 random cardiomyocytes from Lectin stained slides by using a digital microscope $(\times 400)$ and the Image J (version $1.39 \mathrm{u}$ ) software. The total number of cardiomyocytes used for quantification was 230.The Masson's trichrome staining was used to detect fibrosis in heart sections using a Masson's Trichrome stain kit. To determine cytoplasmic vacuolization and myofibrillar loss, we quantified the data by using a scoring method. The extent of histopathology was scored on a scale of $0-3$. If left ventricular heart section showed no signs of myofibrillar loss and cytoplasmic vacuolization, the score was graded as 0 ; slide $<5 \%$ cells exhibiting early myofibrillar loss or cytosolic vacuolization was graded as $1 ; 5-30 \%$ cells exhibiting marked myofibrillar loss and/or cytoplasmic vacuolization was graded as 2 ; diffuse $>30 \%$ cell damage with most exhibiting marked loss-of-contractile elements and myofibrillar disruption was graded as $3^{64,}{ }^{65}$. There were 25 slides per group scored independently for this semi-quantification.

\section{TUNEL staining}

TUNEL (terminal deoxynucleotidyl transferasemediated dUTP nick-end labeling) assessment of myonuclei positive for DNA strand breaks was determined using a fluorescence detection kit (Roche Applied Science, Indianapolis, IN) and fluorescence microscopy. After dewax and rehydrate, paraffin tissue sections were permeabilized with $0.1 \%$ Triton $\mathrm{X}-100$ in $0.1 \%$ sodium citrate for $8 \mathrm{~min}$ on ice. TUNEL reaction mixture containing terminal deoxynucleotidyl transferase $(\mathrm{TdT})$, fluorescein-dUTP was added to the sections in $50-\mu \mathrm{l}$ drops and incubated for $60 \mathrm{~min}$ at $37^{\circ} \mathrm{C}$ in a humidified chamber in the dark. The sections were rinsed three times in PBS for $5 \mathrm{~min}$ each. Following embedding, sections were visualized with an Olympus DP80 microscope equipped with an Olympus MaguaFire SP digital camera. DNase I and label solution were used as positive and negative controls. To determine the percentage of apoptotic cells, the TUNEL-positive nuclei and TUNEL-negative cells were counted using the Image-Pro image analysis software (Media Cybernetics, Bethesda, MD) ${ }^{62}$.

\section{Cardiac lactate measurement}

The cardiac lactate production was determined by using a lactate assay kit (Cell Biolabs, CA, USA). The procedure was performed per the product manual.

\section{Primer design, total RNA extraction, cDNA synthesis, reverse transcription, and real-time polymerase chain reaction}

Total RNA was isolated from left ventricles using the TRIzol reagent (Invitrogen), followed by DNase digestion to eliminate genomic DNA contamination ${ }^{66}$. RNAs were quantified using a NanoDrop ${ }^{\text {TM }} 2000$ spectrophotometer (Thermo Fisher Scientific). Synthesis of cDNA and reverse transcription was performed using $1 \mu \mathrm{g}$ total RNA in a 20- $\mu \mathrm{l}$ system following the instructions of iScript ${ }^{\mathrm{TM}}$ cDNA synthesis kit (Bio-Rad). Ctsk primers located in exon 2 for qPCR were designed by using the PrimerQuest tool on Integrated DNA Technologies' (IDT) website (http://www.idtdna.com/primerquest/home/index). The quantitative real-time PCR was performed for Ctsk, Ctskexon 2, and Rn18s (housekeeping gene) using a C1000 Touch Thermal Cycler CFX96 ${ }^{\text {TM }}$ Real-Time System (BioRad) per the $\mathrm{iQ}^{\mathrm{TM}} \mathrm{SYBR}^{\oplus}$ Green Supermix (Bio-Rad) instructions. Real-time PCR was triplicated for each cDNA sample. The primer sequences are shown in Table 1.

\section{Western blot analysis}

Protein samples were prepared as described previously and separated on SDS-polyacrylamide gels, and transferred electrophoretically to nitrocellulose membranes ${ }^{55}$. The membranes were blocked with $5 \%$ milk in TBS-T, and were incubated overnight at $4{ }^{\circ} \mathrm{C}$ with anti-Cathepsin K (1:500; ab19027), anti-desmin (1:1000; CST5332), antisarcomeric $\alpha$-actinin (1:1000; ab9465), anti-NFkB-p65 (1:1000; CST8242), anti-phospho-IкB $\alpha$ (Ser32/36, 1:500; CST9246), anti- IкB $\alpha$ (1:1000; CST4812), antiinterleukin-1 $\beta$ (IL-1 $\beta, 1: 500 ;$ CST8689), anti- antiinterleukin-6 (IL-6, 1:500; CST12912), anti-TNF- $\alpha$ (1:500, CST3707), anti-phospho-AMPK (Thr172, 1:1000; CST2535), anti-phospho-AKT (Ser473,1:1000; CST4060), anti-cleaved caspase-3 (1:500; CST9661), anti-Bcl-2 (1:1000; CST2870), anti-BAX (1:1000; CST2772), antiGAPDH (loading controls, 1:1000; CST2118) and anti- $\alpha$ tubulin (loading controls, 1:1000; CST2144) antibodies. Blots were incubated with horseradish peroxidase (HRP)conjugated secondary antibody (1:3000; CST7074, CST7076). Antigens were detected by the luminescence method. Band densities were determined using Quantity One software (Bio-Rad, version 4.4.0, ChemiDoc XRS).

\section{Statistical analyses}

The data were presented as mean \pm SEM. Statistical significance $(p<0.05)$ for each variable was estimated by 
an unpaired $t$-test (two-tailed) or a one-way analysis of variance (ANOVA) followed by a Tukey's post hoc analysis.

\section{Acknowledgements}

This publication was made possible by an Institutional Development Award (IDeA) from the National Institute of General Medical Sciences of the National Institutes of Health under Grant \#2P20GM103432. Transgenic mice were generated at the Vector and Transgenic Mouse Core of the University of Washington Diabetes Research Center, P30 DK017047.

\section{Conflict of interest}

The authors declare that they have no conflict of interest.

\section{Publisher's note}

Springer Nature remains neutral with regard to jurisdictional claims in published maps and institutional affiliations.

Supplementary Information accompanies this paper at (https://doi.org/ 10.1038/s41419-018-0727-2).

Received: 12 February 2018 Revised: 19 May 2018 Accepted: 21 May 2018 Published online: 07 June 2018

\section{References}

1. Abdel-Magid, A. F. Inhibition of Cathepsin K: a novel and promising treatment for osteoporosis. ACS Med. Chem. Lett. 6, 628-629 (2015).

2. Lafarge, J. C., Naour, N., Clement, K. \& Guerre-Millo, M. Cathepsins and cystatin C in atherosclerosis and obesity. Biochimie 92, 1580-1586 (2010).

3. Lutgens, S. P., Cleutjens, K. B., Daemen, M. J. \& Heeneman, S. Cathepsin cysteine proteases in cardiovascular disease. FASEB J. 21, 3029-3041 (2007).

4. Wu, H., Du, Q., Dai, Q., Ge, J. \& Cheng, X. Cysteine protease cathepsins in atherosclerotic cardiovascular diseases. J. Atheroscler. Thromb. 25, 111-123 (2017).

5. Zhao, G. et al. Increased circulating Cathepsin $\mathrm{K}$ in patients with chronic heart failure. PLOS ONE 10, e0136093 (2015).

6. Cheng, X. W. et al. Elastolytic Cathepsin induction/activation system exists in myocardium and is upregulated in hypertensive heart failure. Hypertension $\mathbf{4 8}$ 979-987 (2006).

7. Sun, J. et al. Cathepsin $\mathrm{K}$ deficiency reduces elastase perfusion-induced abdominal aortic aneurysms in mice. Arterioscler. Thromb. Vasc. Biol. 32, 15-23 (2012).

8. Hua, Y. et al. Cathepsin K knockout mitigates high-fat diet-induced cardiac hypertrophy and contractile dysfunction. Diabetes 62, 498-509 (2013).

9. Hua, Y. et al. Cathepsin K knockout alleviates pressure overload-induced cardiac hypertrophy. Hypertension 61, 1184-1192 (2013).

10. Hua, Y. et al. Cathepsin $\mathrm{K}$ knockout alleviates aging-induced cardiac dysfunction. Aging Cell 14, 345-351 (2015).

11. Guo, R. et al. Cathepsin $\mathrm{K}$ knockout protects against cardiac dysfunction in diabetic mice. Sci. Rep. 7, 8703 (2017).

12. Chatterjee, K., Zhang, J., Honbo, N. \& Karliner, J. S. Doxorubicin cardiomyopathy. Cardiology 115, 155-162 (2010).

13. Olson, R. D. et al. Regulatory role of glutathione and soluble sulfhydryl groups in the toxicity of adriamycin. J. Pharmacol. Exp. Ther. 215, 450-454 (1980).

14. Powell, S. R. \& Chevion, M. The effect of chronic administration of doxorubicin on the rat cardiac and hepatic glutathione redox system. Res. Commun. Chem. Pathol. Pharmacol. 74, 273-286 (1991).

15. Luo, X. et al. Doxorubicin-induced acute changes in cytotoxic aldehydes, antioxidant status and cardiac function in the rat. Biochim. Biophys. Acta 1360 45-52 (1997)

16. Vassalle, C. \& lervasi, G. Cathepsin K-a classical bone biomarker in cardiovascular disease: the heart is not alone anymore. Atherosclerosis 228, 36-37 (2013).

17. Zhu, W. Q. et al. Acute doxorubicin cardiotoxicity is associated with p53induced inhibition of the mammalian target of rapamycin pathway. Circulation 119, 99-U195 (2009).
18. Xu, X., Bucala, R. \& Ren, J. Macrophage migration inhibitory factor deficiency augments doxorubicin-induced cardiomyopathy. J. Am. Heart Assoc. 2, e000439 (2013).

19. Wang, L. et al. Protection against doxorubicin-induced myocardial dysfunction in mice by cardiac-specific expression of carboxyl terminus of hsp70interacting protein. Sci. Rep. 6, 28399 (2016).

20. Ewer, M. S. \& Ewer, S. M. Cardiotoxicity of anticancer treatments. Nat. Rev. Cardiol. 12, 620 (2015)

21. Billingham, M. E., Mason, J. W., Bristow, M. R. \& Daniels, J. R. Anthracycline cardiomyopathy monitored by morphologic changes. Cancer Treat. Rep. 62 , 865-872 (1978).

22. Jaenke, R. S. Delayed and progressive myocardial lesions after adriamycin administration in the rabbit. Cancer Res. 36, 2958-2966 (1976).

23. Bowles, N. E., Bowles, K. R. \& Towbin, J. A. The "final common pathway" hypothesis and inherited cardiovascular disease. The role of cytoskeletal proteins in dilated cardiomyopathy. Herz 25, 168-175 (2000).

24. Machackova, J., Barta, J. \& Dhalla, N. S. Myofibrillar remodelling in cardiachypertrophy, heart failure and cardiomyopathies. Can. J. Cardiol. 22, 953-968 (2006).

25. Capetanaki, Y. Desmin cytoskeleton in healthy and failing heart. Heart Fail. Rev. 5, 203-220 (2000).

26. Di Somma, S. et al. Desmin-free cardiomyocytes and myocardial dysfunction in end stage heart failure. Eur. J. Heart Fail. 6, 389-398 (2004)

27. Milner, D. J., Weitzer, G., Tran, D., Bradley, A. \& Capetanaki, Y. Disruption of muscle architecture and myocardial degeneration in mice lacking desmin. J. Cell Biol. 134, 1255-1270 (1996).

28. Li, Z. et al. Cardiovascular lesions and skeletal myopathy in mice lacking desmin. Dev. Biol. 175, 362-366 (1996).

29. Sjoblom, B., Salmazo, A. \& Djinovic-Carugo, K. Alpha-actinin structure and regulation. Cell Mol. Life Sci. 65, 2688-2701 (2008).

30. Gupta, V., Discenza, M., Guyon, J. R., Kunkel, L. M. \& Beggs, A. H. alpha-Actinin-2 deficiency results in sarcomeric defects in zebrafish that cannot be rescued by alpha-actinin-3 revealing functional differences between sarcomeric isoforms. FASEB J. 26, 1892-1908 (2012)

31. Mohapatra, B. et al. Mutations in the muscle LIM protein and alpha-actinin-2 genes in dilated cardiomyopathy and endocardial fibroelastosis. Mol. Genet. Metab. 80, 207-215 (2003).

32. Chiu, $C$. et al. Mutations in alpha-actinin-2 cause hypertrophic cardiomyopathy: a genome-wide analysis. J. Am. Coll. Cardiol. 55, 1127-1135 (2010).

33. Maloyan, A., Sayegh, J., Osinska, H., Chua, B. H. \& Robbins, J. Manipulation of death pathways in desmin-related cardiomyopathy. Circ. Res. 106, 1524-1532 (2010).

34. Triplett, J. W. \& Pavalko, F. M. Disruption of alpha-actinin-integrin interactions at focal adhesions renders osteoblasts susceptible to apoptosis. Am. J. Physiol. Cell. Physiol. 291, C909-C921 (2006).

35. Communal, $C$. et al. Functional consequences of caspase activation in cardiac myocytes. Proc. Natl Acad. Sci. USA 99, 6252-6256 (2002).

36. Chen, F., Chang, R., Trivedi, M., Capetanaki, Y. \& Cryns, V. L. Caspase proteolysis of desmin produces a dominant-negative inhibitor of intermediate filaments and promotes apoptosis. J. Biol. Chem. 278, 6848-6853 (2003).

37. Baron, C. P., Jacobsen, S. \& Purslow, P. P. Cleavage of desmin by cysteine proteases: Calpains and Cathepsin B. Meat Sci. 68, 447-456 (2004).

38. Octavia, Y. et al. Doxorubicin-induced cardiomyopathy: from molecular mechanisms to therapeutic strategies. J. Mol. Cell Cardiol. 52, 1213-1225 (2012).

39. Bordoni, A., Biagi, P. \& Hrelia, S. The impairment of essential fatty acid metabolism as a key factor in doxorubicin-induced damage in cultured rat cardiomyocytes. Biochim. Biophys. Acta 1440, 100-106 (1999).

40. Hardie, D. G. Role of AMP-activated protein kinase in the metabolic syndrome and in heart disease. FEBS Lett. 582, 81-89 (2008).

41. Kuznetsov, A. V., Margreiter, R., Amberger, A., Saks, V. \& Grimm, M. Changes in mitochondrial redox state, membrane potential and calcium precede mitochondrial dysfunction in doxorubicin-induced cell death. Biochim. Biophys. Acta 1813, 1144-1152 (2011).

42. Gratia, S. et al. Inhibition of AMPK signalling by doxorubicin: at the crossroads of the cardiac responses to energetic, oxidative, and genotoxic stress. Cardiovasc. Res. 95, 290-299 (2012).

43. Holmgren, G. et al. Identification of novel biomarkers for doxorubicin-induced toxicity in human cardiomyocytes derived from pluripotent stem cells. Toxicology 328, 102-111 (2015). 
44. Swamy, A. V., Gulliaya, S., Thippeswamy, A., Koti, B. C. \& Manjula, D. V. Cardioprotective effect of curcumin against doxorubicin-induced myocardial toxicity in albino rats. Indian J. Pharmacol. 44, 73-77 (2012).

45. Bishop, S. P. \& Altschuld, R. A. Increased glycolytic metabolism in cardiac hypertrophy and congestive failure. Am. J. Physiol. 218, 153-159 (1970).

46. Andreadou, I. et al. Metabonomic identification of novel biomarkers in doxorubicin cardiotoxicity and protective effect of the natural antioxidant oleuropein. NMR Biomed. 22, 585-592 (2009).

47. Ji, C. et al. Exogenous cell-permeable C6 ceramide sensitizes multiple cancer cell lines to doxorubicin-induced apoptosis by promoting AMPK activation and mTORC1 inhibition. Oncogene 29, 6557-6568 (2010).

48. Li, D., Li, J., An, Y., Yang, Y. \& Zhang, S. Q. Doxorubicin-induced apoptosis in H9c2 cardiomyocytes by NF-kappaB dependent PUMA upregulation. Eur. Rev. Med. Pharmacol. Sci. 17, 2323-2329 (2013).

49. Alvarez-Guardia, D. et al. The p65 subunit of NF-kappaB binds to PGC-1alpha, linking inflammation and metabolic disturbances in cardiac cells. Cardiovasc. Res. 87, 449-458 (2010).

50. Kanda, T. \& Takahashi, T. Interleukin-6 and cardiovascular diseases. Jpn. Heart J. 45, 183-193 (2004)

51. Pecoraro, M. et al. Inflammatory mediators in a short-time mouse model of doxorubicin-induced cardiotoxicity. Toxicol. Appl. Pharmacol. 293, 44-52 (2016).

52. Lou, H., Danelisen, I. \& Singal, P. K. Cytokines are not upregulated in adriamycin-induced cardiomyopathy and heart failure. J. Mol. Cell Cardiol. 36 683-690 (2004).

53. Kitamura, Y. et al. Manipulation of Cardiac Phosphatidylinositol 3-Kinase (PI3K)/ Akt signaling by apoptosis regulator through modulating IAP expression (ARIA) regulates cardiomyocyte death during doxorubicin-induced cardiomyopathy. J. Biol. Chem. 289, 2788-2800 (2014).

54. Raymond, C. S. \& Soriano, P. High-efficiency FLP and PhiC31 site-specific recombination in mammalian cells. PLoS ONE 2, e162 (2007).

55. Guo, R. \& Ren, J. Deficiency in AMPK attenuates ethanol-induced cardiac contractile dysfunction through inhibition of autophagosome formation. Cardiovasc. Res. 94, 480-491 (2012).
56. Guo, R., Zhang, Y., Turdi, S. \& Ren, J. Adiponectin knockout accentuates high fat diet-induced obesity and cardiac dysfunction: role of autophagy. Biochim. Biophys. Acta 1832, 1136-1148 (2013)

57. Nguyen, T. V., Jayaraman, A., Quaglino, A. \& Pike, C. J. Androgens selectively protect against apoptosis in hippocampal neurones. J. Neuroendocrinol. 22 1013-1022 (2010)

58. Farivar, T. N., Najafipour, R. \& Johari, P. Nano - drug delivery of apoptosis activator 2 to AGS cells by liposomes conjugated with anti-TROP2 antibody. N. Am. J. Med. Sci. 4, 582-585 (2012).

59. Toldo, S. et al. Comparative cardiac toxicity of anthracyclines in vitro and in vivo in the mouse. PLOS ONE 8, ARTN e5842110.1371/journal.pone.0058421 (2013).

60. Bessho, R. et al. Pyrrolidine dithiocarbamate, a potent inhibitor of nuclear factor kappa-B (Nf-Kappa-B) activation, prevents apoptosis in human promyelocytic leukemia HI-60 cells and thymocytes. Biochem. Pharmacol. 48, 1883-1889 (1994)

61. Schinikerth, V., Bara, A., Mulsch, A. \& Busse, R. Pyrrolidine dithiocarbamate selectively prevents the expression of the inducible nitric-oxide synthase in the rat aorta. Eur. J. Pharmacol. 265, 83-87 (1994).

62. Guo, R. \& Ren, J. Alcohol dehydrogenase accentuates ethanol-induced myocardial dysfunction and mitochondrial damage in mice: role of mitochondrial death pathway. PLoS ONE 5, e8757 (2010).

63. Wang, D. et al. Cardiomyocyte cyclooxygenase-2 influences cardiac rhythm and function. Proc. Natl Acad. Sci. USA 106, 7548-7552 (2009).

64. Carvalho, R. A. et al. Metabolic remodeling associated with subchronic doxorubicin cardiomyopathy. Toxicology 270, 92-98 (2010).

65. Singla, D. K., Ahmed, A., Singla, R. \& Yan, B. B. Embryonic stem cells improve cardiac function in doxorubicin-induced cardiomyopathy mediated through multiple mechanisms. Cell Transplant. 21, 1919-1930 (2012).

66. Dong, F., Li, Q., Sreejayan, N., Nunn, J. M. \& Ren, J. Metallothionein prevents high-fat diet induced cardiac contractile dysfunction: role of peroxisome proliferator activated receptor gamma coactivator 1 alpha and mitochondrial biogenesis. Diabetes 56, 2201-2212 (2007). 